\title{
IDENTIFICATION OF CONSTITUTIVE RELATIONSHIPS FOR CONCRETE USING DISCRETE AND FINITE ELEMENT FRAMEWORK
}

\author{
FREDERIC RAGUENEAU ${ }^{*}$, MAXIME VASSAUX ${ }^{*}$ CÉCILE OLIVER-LEBLOND ${ }^{*}$ AND \\ BENJAMIN RICHARD ${ }^{\dagger}$ \\ LMT, ENS-Cachan/CNRS/Univ. Paris-Saclay \\ Cachan, F-94235, FRANCE \\ e-mail: ragueneau@lmt.ens-cachan.fr \\ LMT, ENS-Cachan/CNRS/Univ. Paris-Saclay \\ Cachan, F-94235, FRANCE \\ e-mail: vassaux@1mt.ens-cachan.fr \\ LMT, ENS-Cachan/CNRS/Univ. Paris-Saclay \\ Cachan, F-94235, FRANCE \\ e-mail: coliver@1mt.ens-cachan.fr \\ * LMT, ENS-Cachan/CNRS/Univ. Paris-Saclay \\ Cachan, F-94235, FRANCE \\ e-mail: coliver@1mt.ens-cachan.fr \\ †CEA, DEN, DANS, DM2S, SEMT, Laboratoire d'Etudes de Mécanique Sismique \\ Gif-sur-Yvette, F-91191, FRANCE \\ e-mail: Benjamin.RICHARD@cea.fr
}

Key words: Concrete, constitutive equation, discrete element, cyclic loading

\begin{abstract}
Considering different scales and phenomena, the models used to describe the cracked behavior of concrete cannot be of the same type. The aim of this contribution is to present different tools, used at their relevant physical scales as well as their mutual enhancement for a global structural approach. Such an issue is greatly emphasized when dealing with cyclic and dynamic loadings, involving complex stress paths. A discrete element model (DEM) is presented, introducing within an implicit framework, local nonlinear mechanisms such as brittle failure, contact, frictional sliding and scale effects can be taken into account. Such a local model will help one, through virtual testing procedures to express constitutive laws allowing for a refine description at the representative volume element (RVE) level of constitutive relationship based on coupled plasticity-damage models. Based on a structural case-study, the relevancy of the full analysis chain is presented.
\end{abstract}

\section{INTRODUCTION}

Sustainability issues of concrete structures are mainly linked to their durability and water tightness capabilities. These features are evolving along with time due to cracking of concrete which is an unavoidable behavior regarding the design of such structures. Cracking has two main consequences: at the microscale, the permeability and diffusion 
processes directly depend on crack's opening, spacing and tortuosity whereas at the macroscale, the homogenized degradation of the constitutive material leads to stiffness loss, modification of the internal forces distribution and loss of the load bearing capacities. Considering those different scales and phenomena, the models used to describe the cracked behavior of concrete cannot be of the same type. The aim of this contribution is to present different tools, used at their relevant physical scales as well as their mutual enhancement for a global structural approach. Such an issue is greatly emphasized when dealing with cyclic and dynamic loadings, involving complex stress paths.

A discrete element model (DEM) is presented, introducing within an implicit framework, local nonlinear mechanisms such as brittle failure, contact, frictional sliding and scale effects. Such a local model will help one, through virtual testing procedures to express constitutive laws allowing for a refine description at the representative volume element (RVE for Finite Element Technique) level of constitutive relationship based on coupled plasticity-damage models. Original developments will be presented accounting for regularized crack effects on cyclic behavior of brittle materials. Structural computations at the large-scale civil engineering structural scales are robustly achieved using this model. Based on a structural case-study, the relevancy of the full analysis chain is presented as conclusions of this contribution.

\section{DISCRETE CRACKING AND CYCLIC BEHAVIOR}

This section is devoted to the recent development performed within the framework of discrete element models $[1,2,3]$ in order to account for physical features such as unilateral crack behavior and frictional sliding. The numerical implementation is realized through implicit algorithms allowing efficient and robust virtual tests.

\subsection{Discrete element model formulation: cohesive forces}

In this contribution, the combined beamparticle model - inspired by D'Addetta et al. [4] and modified by Delaplace [5] and Vassaux et al. [6] is used. The concrete is represented through polygonal particles linked together by brittle beams and exhibiting frictional contact after failure of the beams. With this model, it is possible to qualitatively reproduce the failure pattern for compressive or tensile simulations.

In order to represent the cohesion of the material, the particles are joined together through Euler-Bernoulli beams linking the centroid of the particles. Those particles are rigid. Therefore, the cohesive forces must reproduce the elastic behavior of the material. The beam $i-j$, linking the particles $i$ and $j$, has four parameters (see on figure 1). Two of them, the length $l_{b, i j}$ and the cross section area $A_{b, i j}$, are prescribed by the mesh geometry and are different for each beam. The two other parameters, the young modulus of the beams $E$ and the coefficient of inertia $\alpha=64 \pi / A_{b, i j}^{2}$, where $I_{b, i j}$ is the moment of inertia, are supposed equal for all beams.

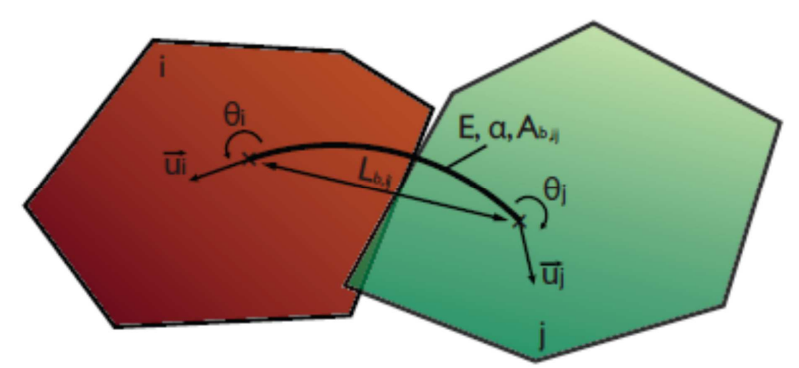

Figure 1: Cohesion between particles

The cohesion forces and moment between two connected particles $i$ and $j$ are expressed as: 


$$
F_{c o h, i j}=\left\{\begin{array}{c}
F_{N, i j}=\frac{E A_{b, i j}}{l_{b, i j}}\left(u_{i}-u_{j}\right) \cdot n_{b, i j} \\
F_{T, i j}=\frac{12 E I_{b, i j}}{l_{b, i j}^{3}}\left(u_{i}-u_{j}\right) \cdot t_{b, i j}-\frac{6 E I_{b, i j}}{l_{b, i j}^{2}}\left(\theta_{i}-\theta_{j}\right) \\
M_{Z, i j}=\frac{6 E I_{b, i j}}{l_{b, i j}^{2}}\left(u_{j}-u_{i}\right) \cdot t_{b, i j}+\frac{4 E I_{b, i j}}{l_{b, i j}}\left(\theta_{i}-\frac{\theta_{j}}{2}\right)
\end{array}\right.
$$

where $n_{b, i j}$ and $t_{b, i j}$ are the normal and tangential vectors of the cross-section of the beam $i-j$. At this point, the discrete model is equivalent to a lattice model $[7,8]$.

\subsection{Frictional contact}

The modelling of concrete failure under cyclic or multi-axial loadings requires to consider frictional contact interactions between particles to capture mechanisms such as crack closure or aggregate interlocking. Contact interactions are only introduced when two separated particles - i.e. that are not linked by a cohesive beam - overlap. The magnitude of the contact forces, as well as their direction and their point of application, is computed as a function of the overlapping areas because particles are perfectly rigid bodies.

First, it is necessary to detect the overlapping of two particles. Since particles are polygonal, the intersection cannot be described analytically as with discs and numerical tools are required. Overlap detection is a time-consuming process, increasing quickly with regard to the number of particles. In order to limit its costs, intersection search is restricted to close neighbors [9]. The complete description of the polygonal intersection is computed through an algorithm developed for convex polygons [10].

The beam theory used for cohesion reveals to be very convenient for elastic contact force computation between polygonal particles [11, 4]. A slight modification is made in the normal force formula to introduce the overlap area $\mathrm{Sr}$ :

$$
F_{c o n t, i j}=\frac{E S_{r, i j}}{l_{c, i j}} n_{c, i j}
$$

where $l c$ is a characteristic length supposed to be an average of the equivalent diameter (implying an equal surface for a virtual round particle) of the particles $\left(D_{i}\right.$ and $\left.D_{j}\right)$.

$$
\frac{1}{l_{c}}=\frac{1}{2}\left(\frac{1}{D_{i}}+\frac{1}{D_{j}}\right)
$$

A Coulomb's type of friction is considered introducing the friction coefficient $\mu$ :

$$
\begin{aligned}
& F_{\text {fric }, i j} \\
& =\min \left(\frac { E I _ { c , i j } } { l _ { c , i j } ^ { 3 } } \left(\left(u_{c, i j}-u_{c, i j}\right) \cdot t_{c, i j}\right.\right. \\
& \left.\left.-\Delta u_{s, i j}\right), \mu\left\|F_{\text {cont }, i j}\right\|\right)
\end{aligned}
$$

where the moment of inertia is $I_{c}=\frac{1 \times l_{c}^{3}}{12}$

\subsection{Energy dissipation and rupture threshold}

In order to reproduce the post-peak behaviour in tension, we introduce a statistical distribution of the breaking parameters. Gauss and Weibull distributions have been proposed and investigated. Morice [12] showed that the resulting displacement field at the top of the crack is visually the same as long as the distributions are identical in terms of mean value and standard deviation. However, following Van Mier et al. [8] conclusions regarding the crack patterns, a Weibull distribution has been chosen. The Weibull probability density function is:

$$
f(x)=\frac{k}{\lambda}\left(\frac{x}{\lambda}\right)^{k-1} e^{-\left(\frac{x}{\lambda}\right)^{k}}
$$

with $\lambda$ the scale factor and $\mathrm{k}$ the shape factor of the Weibull distribution. We consider that the spatial variability is the same for both breaking parameters. Consequently the shape factor is kept identical for each parameter and only the scale factor varies. Moreover, no minimal or maximal value has been assigned to the breaking parameters contrary to the proposition of Van Mier et al. [8]. Thus, the number of parameters is minimized. 
Many formulations of failure criterion can already be found in the literature [13] and some of them offer an accurate description of the complete failure behaviour. Vassaux et al. [14] tested several formulations based on Rankine or Mohr-Coulomb criterion. They observed that the use of extensions and rotations instead of axial forces and bending moments, as originally proposed by Schlangen and Van Mier [4], do not change the results. Therefore, a criterion based on $\varepsilon_{i j}, \theta i$ and $\theta j$ is chosen to stay in the kinematic framework of this beam-particle model. The formulation can be seen as a Mohr-Coulomb-type criterion controlled with only two parameters, setting for each beam the cone vertex position $\varepsilon_{c r, i j}$ and the intersection of its edge with the zero-extension plane $\theta_{c r, i j}$. The resistance in rotation increases with the level of contraction inside the beam. Such formulation of the failure criterion can be seen as an homogenization of lower scale mechanisms that are not considered in the model, especially porosity compaction.

$P_{i j}=\frac{\varepsilon_{i j}}{\varepsilon_{i j}^{c r}}+\frac{\left|\theta_{i}-\theta_{j}\right|}{\theta_{i j}^{c r}}>1$

The figure 6 allows one to figure out the failure threshold representation.

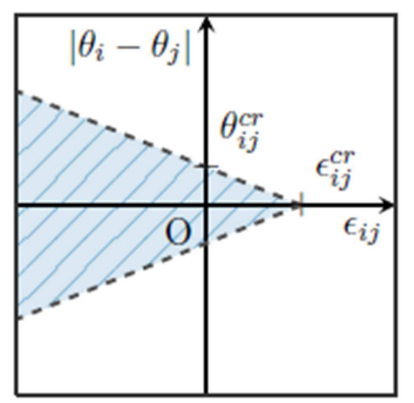

Figure 2: Mohr-Coulomb failure criterion

\subsection{Identification and validation}

Identification procedures concerning the element size, elasticity parameters, failure and friction can be found in [14] allowing to reproduce strong nonlinear behavior under usual compressive loadings as demonstrated in figure 3 considering different levels of adherence between the sample and the loadings platens. Experimentally [15], the inclination of the localized macroscopic crack has been observed to depend on the adherence level of the platens. The beam-particle model with the modification to apply partially restrained boundary conditions allows the reproduction of the experimental results: the crack pattern slope increases with the friction.

The shear test proposed by Fenwick and Paulay [16] allows testing a concrete specimen under pure mode II failure. The small specimen ( $0.45 \mathrm{~m}$ long and $0.1 \mathrm{~m}$ high) is first loaded under uni-axial tension to form a localized crack in between two small notches at mid-length of the sample. Then the specimen is maintained to keep the crack opening constant, and shear load is applied.

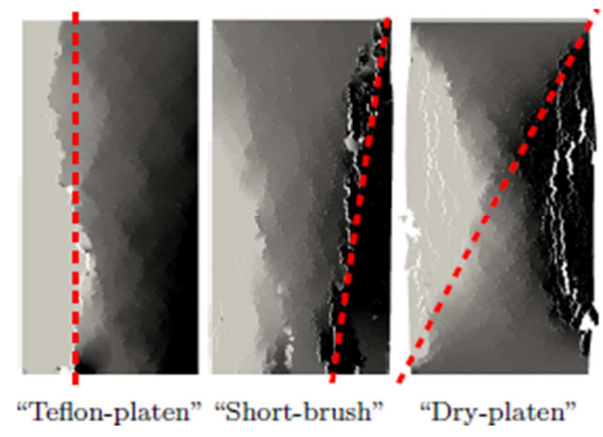

Figure 3: Influence of the adherence of the loaded ends on the cracking patterns in compression.

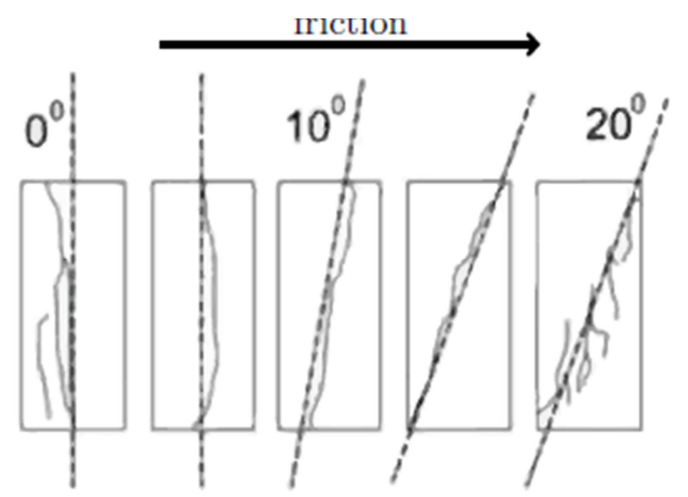

Figure 4: Experimental crack orientation considering platen friction [15] 
The evolution of the shear stress with the shear displacement for several crack widths i.e. different confinement levels - is experimentally available [17]. The critical extension scale factor is re-calibrated in order to fit the experimental response for the smallest crack-width $(c w=0.00006 \mathrm{~m})$. The whole parameter set is given in the table 1 .

Table 1: Parameters values

\begin{tabular}{lllllll}
\hline$l_{p}(m)$ & $\alpha$ & $\begin{array}{l}\mathrm{E} \\
(\mathrm{GPa})\end{array}$ & $\lambda_{\varepsilon_{c r}}$ & $\theta_{\varepsilon_{c r}}$ & $\mathrm{k}$ & $\mu$ \\
\hline 0.002 & 0.83 & 46 & 4.35 & 3.26 & 2.8 & 0.7 \\
& & & $10^{-4}$ & $10^{-3}$ & & \\
\hline
\end{tabular}

The model is able to reproduce not only the peak-load values but also the pre-peak overall slope of the smooth experimental curves (see on figure 5). Note that the offsets in the prepeak behaviour (horizontal slope), especially visible at high confinements, are evidences of the "aggregate interlocking" mechanism. Shear stresses transit through the crack because of geometrical incompatibilities, until particles at the crack interface start to spall off, once all their cohesive beams are broken. Sliding is then initiated at crack lips until new incompatibilities are generated. The model would hardly capture such mechanism without the use of polygonal particles.

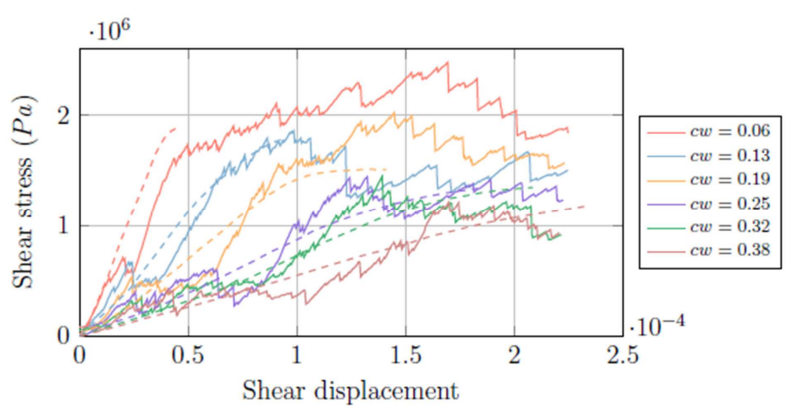

Figure 5: Response of the model for Fenwick and Paulay shear test

\section{CONTINUOUS MODELLING}

The design of robust and accurate constitutive models for quasi-brittle materials accounting for cyclic loading effects is an essential step in the process of predicting the response of civil engineering structures under complex loading, and more specifically earthquakes. Several modelling techniques have been developed at the macroscopic scale to depict quasi-brittle materials behavior, based on empirical models $[18,19,20]$, micromechanical models $[21,22]$ or phenomenological models [23, 24, 25, 26, 27].

Toward the completion of the simulation of three-dimensional structures made of concrete subjected to cyclic loading, phenomenological models have inherent advantages. However, the constitutive equations complexity increases rapidly when trying to capture consequences of mechanisms induced under uniaxial cyclic loading. Indeed even recently developed models are either not robust enough to simulate the complete response of structures subjected to cyclic loading, or do not reproduce accurately phenomena related to cracks closure and friction, as outlined by results of the ConCrack international benchmark.

The first objective of this contribution is to enhance the efficiency of the model proposed in [28] to achieve structural simulations. A specific attention is paid to phenomena observed under cyclic loading. Issues encountered with the current model are partly induced by a lack of experimental data, preventing from establishing a finer mechanical description of the material's behaviour. Experimental data on quasi-brittle materials Representative Volume Element (RVE) subjected to reverse cyclic loading are sparse due to control and repeatability issues. Therefore the replacement of part of laboratory experimentation by virtual testing is investigated. A RVE is considered here to be approximately of a $0.1 \mathrm{~m}$ characteristic length, such as the material can be considered homogeneous with respect to different phases.

\subsection{Thermodynamic formulation}

The phenomenological macroscopic model is formulated using a rather classic decomposition of the total stress in the RVE. It is considered that the total stress $\boldsymbol{\sigma}$ can be split in two independent parts: 


$$
\boldsymbol{\sigma}=\boldsymbol{\sigma}^{m}+\boldsymbol{\sigma}^{f}
$$

with the stress in the cracked continuous media $\boldsymbol{\sigma}^{m}$, neglecting any interaction between the cracks, classically modeled with a damage model; and the stress in the cracks when closed $\boldsymbol{\sigma}^{f}$. Free energies $\Psi^{f}$ and $\Psi^{m}$ respectively associated to the two stress tensors are defined, and compose the total free energy of the specimen:

$$
\Psi=\Psi^{m}+\Psi^{f}
$$

\subsection{Cracking modelling}

Fracture processes are modeled by means of the continuum damage theory. The simpler the damage variable is kept, the more robust the proposed macroscopic model is. Therefore, in view of the structural applications of the proposed model, an isotropic damage model is formulated, implying a unique scalar damage variable. The free energy associated to the cracked continuous media simply writes:

$$
\Psi^{m}=\frac{1}{2}(1-D) \boldsymbol{\epsilon}: \boldsymbol{C}: \boldsymbol{\epsilon}+\Psi^{m, D}(z)
$$

with $D$ the isotropic damage variable, $\boldsymbol{\epsilon}$ the second-order total strain tensor, $\boldsymbol{C}$ the fourthorder Hooke's tensor, $Z$ the isotropic hardening variable, and $\Psi^{m, D}$ the free energy related to damage. The formulation of the nonassociated pseudo-potential of dissipation $\varphi^{m}$ is based on the Mazars failure criterion and expressed in terms of thermodynamic variables.

$$
\varphi^{m}=\bar{Y}-\left(Y_{0}+Z\right)
$$

where $\mathrm{Z}$ stands for the thermodynamic force associated to $\mathrm{z}, \bar{Y}$ the energy rate $\bar{Y}=\frac{1}{2} E \varepsilon_{0} \varepsilon_{e q}$ which is written as a function of Mazars equivalent strain $\varepsilon_{e q}=\sqrt{\langle\epsilon\rangle_{+}:\langle\epsilon\rangle_{+}}, \varepsilon_{0}$ the elastic limit strain, and $Y_{0}$ the elastic limit energy rate written in a similar fashion

$$
Y_{0}=\frac{1}{2} E \varepsilon_{0} \varepsilon_{0}
$$

The asymmetry between traction and compression loading is only considered through its consequence on the peak load and the softening behavior of the material, and is introduced in the damage variable evolution law derived from $\Psi^{m, D}$. This leads to a damage evolution law expressed as:

$$
D=1-\frac{Y_{0}}{\bar{Y}} \exp \left[-\frac{B_{0}}{\kappa}\left(\bar{Y}-Y_{0}\right)\right]
$$

where $B_{0}$ stands for a parameter controlling the softening behavior and $\kappa$ computed as follows:

$$
\kappa=1+k_{0}\left(\frac{\langle\boldsymbol{C}: \boldsymbol{\epsilon}\rangle_{-}:\langle\boldsymbol{C}: \boldsymbol{\epsilon}\rangle_{-}}{\langle\boldsymbol{C}: \boldsymbol{\epsilon}\rangle^{1 / 2}\langle\boldsymbol{C}: \boldsymbol{\epsilon}\rangle}\right)^{1 / 2}
$$

where $k_{0}$ stands for a parameter measuring the influence of the confining pressure, and therefore only influences the failure behavior when cracks are induced indirectly (e.g. in compression).

\subsection{Stiffness recovery}

Cracks mechanical behavior described by the stress tensor $\boldsymbol{\sigma}^{f}$ is first considered elastic. $\boldsymbol{\sigma}^{f}$ is defined as non-linear function of the strain tensor $\boldsymbol{\varepsilon}^{f}$, which could be called the homogenized contribution of cracks opening to the total strain of the RVE. The following assumption is made on the evolution of $\boldsymbol{\sigma}^{f}$ with respect to $\boldsymbol{\varepsilon}^{f}$ :

$$
\dot{\boldsymbol{\sigma}}^{f}=\vartheta\left(\dot{\boldsymbol{\varepsilon}}^{f}\right) \boldsymbol{C}: \dot{\boldsymbol{\varepsilon}}^{f}
$$

The function $\vartheta$ is scalar, in other words, the tangent modulus of the cracks stress-strain relationship is proportional to the undamaged Hooke's elastic tensor. $\vartheta$ represents the part of the lost stiffness due to cracking which is recovered thanks to cracks closure, and can only take values ranging from 0 , when cracks are completely opened, to 1 , when cracks are completely closed. Since $\vartheta$ evolves according to the materials solicitation, it is considered to 
be dependent on $\dot{\boldsymbol{\varepsilon}}^{f}$. The elastic part of the free energy associated to cracks behavior is then written as:

$$
\Psi f, e=\int\left(\int \vartheta\left(\boldsymbol{\varepsilon}^{f}\right) \boldsymbol{C}: d \boldsymbol{\varepsilon}^{f}\right) d \boldsymbol{\varepsilon}^{f}
$$

\subsection{Frictional sliding}

The explanation of hysteresis effects relying on frictional sliding occurring at the cracks surfaces justifies a modeling method based on plasticity theory. In consequence, a perfect plasticity model along with a DruckerPrager criterion is utilized. Because of perfect plasticity, the free energy $\Psi^{f}$ is reduced to an elastic part $\Psi^{f, e}$ and introduces a single internal variable, the plastic strain accumulated through sliding between the cracks $\boldsymbol{\varepsilon}^{f, p}$, defined as $\boldsymbol{\varepsilon}^{f}=\boldsymbol{\varepsilon}^{f, p}+\boldsymbol{\varepsilon}^{f, e}$. Considering the assumption of isochoric transformation for $\boldsymbol{\varepsilon}^{f, p}, \Psi^{f}$ sums up as:

$\Psi^{f}=\int\left(\int \vartheta\left(\boldsymbol{\varepsilon}^{f}\right) \boldsymbol{C}: d \boldsymbol{\varepsilon}^{f}\right) d \boldsymbol{\varepsilon}^{f}-\frac{1}{2} \vartheta\left(\boldsymbol{\varepsilon}^{f}\right) \boldsymbol{\varepsilon}^{f, p}: \boldsymbol{C}: \boldsymbol{\varepsilon}^{f, p}$

Conditions of continuity of the free energy $\Psi^{f}$ are not changed when introducing frictional sliding. The pseudo-potential of dissipation is a Drucker-Prager criterion:

$$
\varphi^{f}=\sqrt{J_{2}\left(\boldsymbol{\sigma}^{f}\right)}+\mu_{0} I_{1}\left(\boldsymbol{\sigma}^{f}\right)
$$

where $\mu_{0}$ stands for a parameter which could be assimilated to a friction coefficient. Regarding the physical significance of the chosen criterion, the $J_{2}$ part refers directly to shear occurring in the cracks, while $I_{1}$ rather refers to cracks surface normal pressure. In consequence, when $J_{2}$ exceeds $\mu_{0} I_{1}$, frictional sliding is observed. Furthermore, the $J_{2}$ part depends on $\vartheta$ through $\boldsymbol{\sigma}^{f}$, therefore depends on the proportion of closed cracks. Thus when all the cracks are open the $J_{2}$ is negligible and as expected no frictional sliding is observed.

\section{IDENTIFICATION AND VIRTUAL TESTING}

\subsection{Unilateral effect}

The formulation of the continuum model accounting for cyclic effects has almost fully been presented, only remains the function $\vartheta$ to be defined. Its physical sense has already been explained, namely the evolution of the proportion of closed cracks with respect to the cracks strain tensor $\boldsymbol{\varepsilon}^{f}$. However, $\vartheta$ remains to be characterised. This process is undertaken using the virtual testing machine aforementioned and the discrete simulations of the uni-axial cyclic test.

The function $\vartheta$, as the proportion of closed cracks, represents the proportion of cracks in which forces transit and contribute to the stiffness recovery of the specimen. The evolution of $\vartheta$ is characterized analyzing the evolution of the ratio of number of contacts detected and the number of cracks initiated in the virtual material sample during the simulation of the uni-axial cyclic test. The analysis is led for different damage levels, that is different maximal cracks strains of $\varepsilon_{\text {max }}^{f}$. The figure 6 shows the evolution of the proportion of closed cracks during unloading phases.

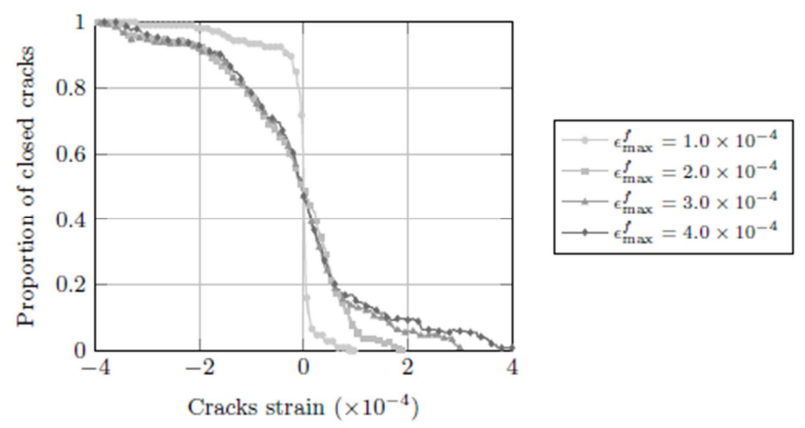

Figure 6: Dependency of the proportion of closed cracks to $\varepsilon_{\max }^{f}$

Independently of of $\varepsilon_{\max }^{f}$, the proportion of closed cracks follows a sigmoidal evolution with respect to $\varepsilon^{f}$. To determine an analytic expression for $\vartheta$ let us consider the probabilistic event "a crack closes", $\vartheta$ is the 
distribution function of this event. From the results obtained with the microscopic model, it appears that this event follows a symmetrical distribution centered in $\varepsilon^{f}=0$, therefore it could be assumed that the event "a crack closes" follows a Gaussian distribution of zero mean. The maximal cracks strain $\varepsilon_{\max }^{f}$ affects the evolution of the proportion of closed cracks. The more damaged the specimen, the bigger the variance of the event "a crack closes". The function $\vartheta$ is finally chosen to account for this dependency:

$$
\vartheta=1-\frac{1}{1+\exp \left[-\frac{\alpha_{0}}{I_{1}\left(\varepsilon_{\text {max }}^{f}\right)} I_{1}\left(\varepsilon^{f}\right)\right]}
$$

where $\alpha_{0}$ stands for a parameter controlling a reference variance of the event a cracks closes".

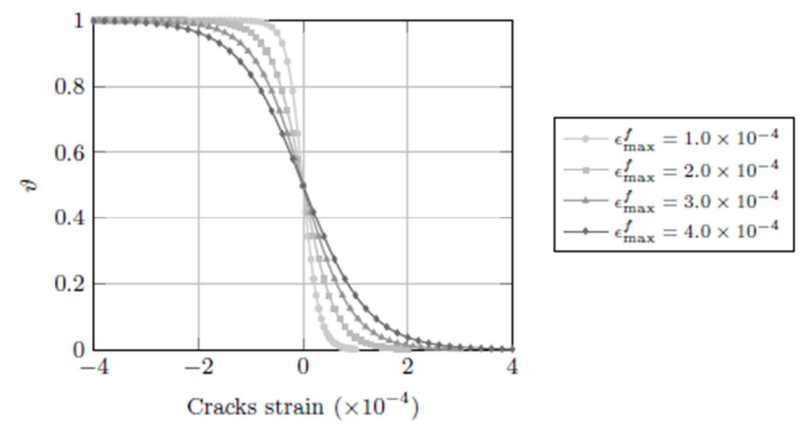

Figure 7: Dependency of the proportion of closed cracks to $\varepsilon_{\max }^{f}$ evaluated with $\alpha_{0}=6.5$

\subsection{Frictional sliding identification}

By means of the microscopic model it is possible to estimate the dissipated energy specific to frictional sliding, among other dissipation mechanisms. Therefore, the parameter $\mu_{0}$ is calibrated in order to observe an identical friction specific dissipation in concrete RVEs modelled using the microscopic model and the macroscopic continuum model (i.e a Gauss point).

The comparison of both models is realized during the simulation of a complete cycle of uni-axial cyclic test, namely loading, unloading, reloading. The amplitude of the cycle is arbitrarily chosen to vary from $\varepsilon_{\text {max }}^{f}=2 . \times 10^{-4}$ to $\varepsilon_{\text {max }}^{f}=-1 . \times 10^{-4}$ back to $\varepsilon_{\max }^{f}=2 . \times 10^{-4}$. Such amplitudes correspond to strains where the most dissipation through friction, and therefore hysteresis effects, is expected due to cracks opening and closure.

Evolutions of the computed energies during the aforementioned loading cycle are presented in figure 8 . Both dissipated energies remain null during the loading step, cracks are completely opened. Then, an important increase is observed during unloading, while cracks progressively close. During reloading, both energies evolutions stagnate first since cracks are completely closed, before opening progressively leading to another increase of the dissipated energy related to friction.

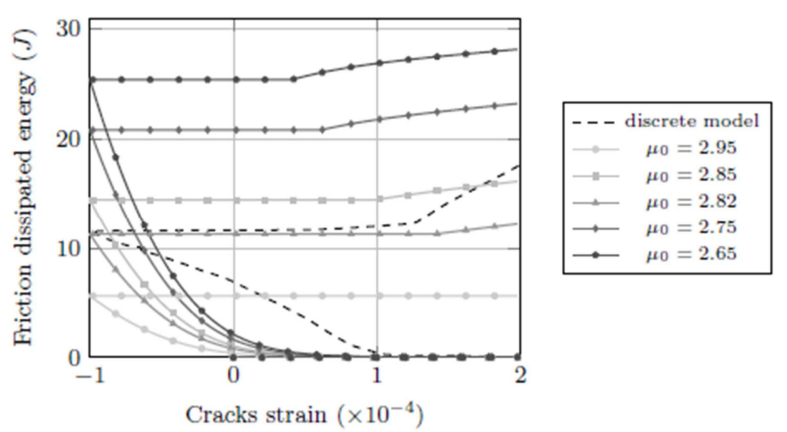

Figure 8: Sensitivity of the friction related dissipated energy of the continuum model to the parameter $\mu_{0}$

The parameter $\mu_{0}$ is then evaluated at $\mu_{0}=2.82$. It might be added that the friction related dissipated energies computed with both models present similar trends, which comforts the choice of the perfect plastic modelling, along with a Drucker-Prager criterion, of the cracks frictional sliding mechanism.

\section{VALIDATION AND CASE-STUDY}

\subsection{Local responses}

Parameters of the continuum medium part of the model are calibrated by equivalence with macroscopic reference results provided by the virtual testing machine on a square sample of $0.1 \mathrm{~m}$ side length. The tension resistance, the tension fracture energy, and the compression resistance are respectively utilized to 
calibrate $\varepsilon_{0}, B_{0}$ and $k_{0}$. In the whole section results plotted with dashed lines refer to the results provided by the virtual testing machine, and plain lines refer to results obtained with the continuum model.

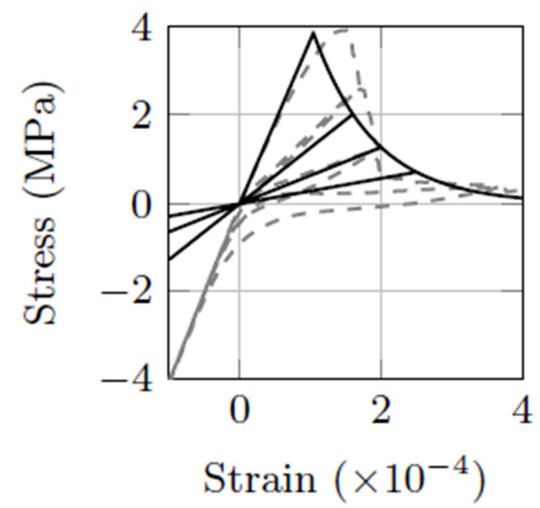

Figure 9: Cyclic response without cracks behaviour

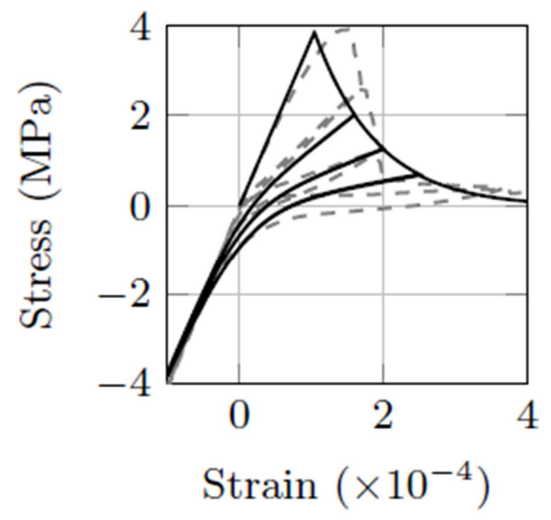

Figure 10: Cyclic response with elastic cracks behaviour

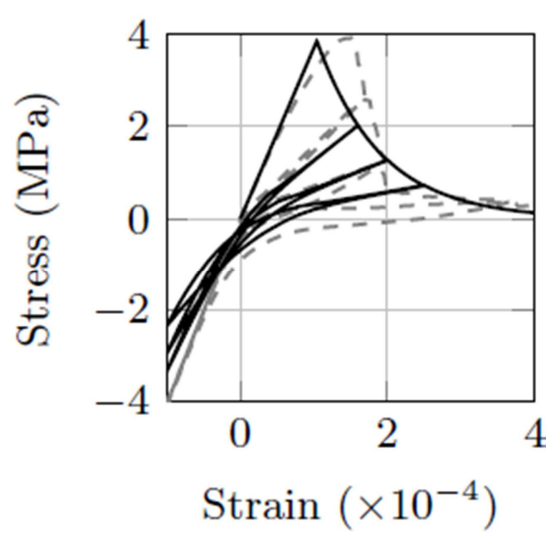

Figure 11: Cyclic response with elasto-plastic cracks behaviour

The uni-axial reverse cyclic test response obtained with the macroscopic model is fairly close to the reference response obtained with the virtual testing machine. First, the addition of the non-linear model of cracks behavior to a classic model, utilized to describe continuum medium's behavior, allows reproducing the progressive stiffness recovery as well as residual strains which disappear in compression. Second, the addition of the plastic model of cracks behavior, enables the emergence of the hysteretic behavior, crescentshaped hysteresis loops are observed at accurate stress levels. Thus, dissipative mechanisms are activated for appropriate solicitations amplitudes.

\subsection{Structural case-study}

In this section, the constitutive law exposed in this paper is used to assess the dynamic behavior of a large scale RC structure subjected to earthquake loading. This RC specimen has been tested by means of shaking table tests within the framework of a wide research project titled Seismic design and bestestimate Methods Assessment for Reinforced concrete buildings subjected to Torsion and non-linear effects (SMART) [29], supported by Electricite de France (EDF) and the French Alternative Energies and Atomic Energy Commission (CEA) under the hospice of the International Atomic Energy Agency (IAEA). One of the objectives of this project was to provide the international earthquake engineering community with a set of welldocumented data in order to help validating the numerical nonlinear constitutive laws. To reach this objective, two experimental campaigns have been carried out on the AZALEE shaking.

The RC specimen (figure 12) is a scaled model of a simplified half part of a nuclear electrical building. It has been prepared to reproduce the geometrical, physical and dynamical characteristics of a part of the real building. Due to the inherent limitations related to the laboratory's capacity, regardless of the laboratory in question, some simplifying assumptions have to be considered. The model had to be geometrically reduced to a scale equal to $1 / 4$. 
The SMART 2013 structure has been meshed by means of eight-node solid elements. The reinforcing steel bars (both longitudinal and stirrups) have been described by means of truss elements. In addition, the shaking table has also been included in the structural model to ensure a satisfactory description of the boundary conditions. As this way, the seismic loading can be imposed by prescribing the displacement time histories at anchorage points of the actuators. The meshes of anchorage of the actuator.

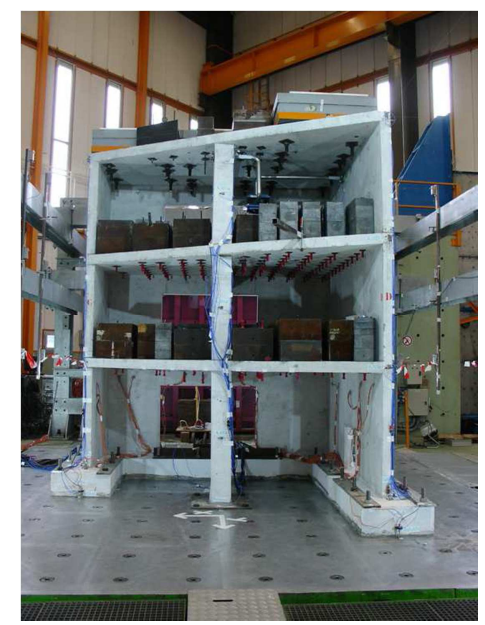

Figure 12: Picture of the structural specimen

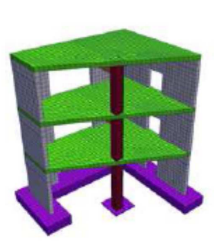

(a) Concrete

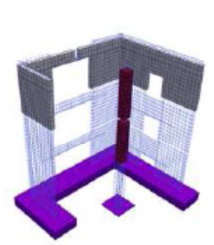

(b) Steel

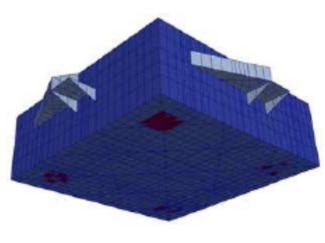

(c) Shaking table
Figure 13: Finite Element mesh of the complete dynamic system

The SMART 2013 structure has been meshed by means of eight-node solid elements. The reinforcing steel bars (both longitudinal and stirrups) have been described by means of truss elements. In addition, the shaking table has also been included in the structural model to ensure a satisfactory description of the boundary conditions. As this way, the seismic loading can be imposed by prescribing the displacement time histories at anchorage points of the actuators. The meshes of the concrete, steel and shaking table are shown in figures 13 .

Three types of signals have been considered in the SMART 2013 experimental campaign: the design signal (with a PGA equal to $0.2 \mathrm{~g}$ ), the main shock of the Northridge earthquake (with a PGA equal to $1.78 \mathrm{~g}$ ) which took place in California, USA, in 1994 and the first aftershock related to the same earthquake (with a PGA equal to $0.33 \mathrm{~g}$ ) recorded at the same monitoring station as the one chosen for the main shock signals. In this study, to choice to analyze the design signal has been made. Indeed, this loading induces only concrete non-linearities which should be taken account in order to describe accurately the experimental behavior of the specimen. It is important to ensure, for validating purposes, that the energy dissipates only in the concrete and not in the steel since the aim of this structural case is to assess the relevancy of the concrete constitutive law. Therefore, this loading can be considered as discriminant.

Two cases have been considered in order to show the importance to take the frictional sliding mechanism into account. In the first case, the increment of the crack plastic strain is set up to zero whereas in a second case, it is computed according the constitutive equations presented here above. As this way, it is possible to assess the influence of this dissipative mechanism on the results obtained at the member scale. For both cases, a Rayleigh viscous damping model has been included. The related parameters are the same and they have been calibrated considered the first and the third eigenfrequency of the dynamic system with a damping ratio equal to $2 \%$. This equivalent viscous matrix allows accounting for damping at low level of excitations implying only a linear behavior of the materials. The results obtained at the first floor are presented in figures 14 .

Thanks to regularized crack closing model, robust computations, in a 3D solid elements description, without encountering classical convergence issues when dealing with nonsmooth unilateral effect. The introduction of frictional sliding during crack closing seems to improve the predictivity of the model. But the 
ratio (and its evolution during loading) of the dissipated energy between the material nonlinearity and the global damping matrix is still an undergoing work.
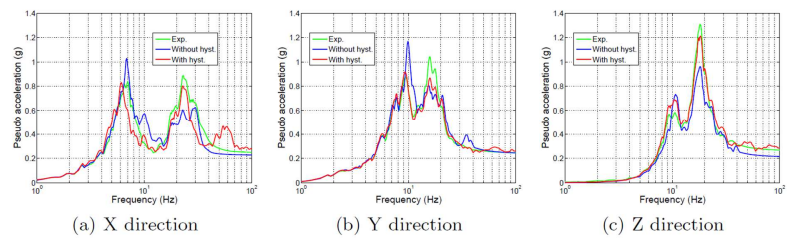

Figure 14: Comparison of the results with and witout hysteretic effects - response spectra at the first floor

\section{CONCLUSIONS}

The purpose of the present paper was to propose a two-level description of concrete cracking subject to cyclic loading. A microscopic model based on the discrete modelling approach is proposed allowing to account for refine phenomena such as crack opening, size effect, crack closure, frictional sliding. A continuum model for quasi-brittle materials able to reproduce phenomena observed under cyclic loading, while remaining sufficiently robust to simulate the behavior of massive structures is also proposed. The formulation of macroscopic constitutive laws has been established using a virtual testing approach. The continuity of the model (under reverse loading) has been tackled introducing the distribution function of a statistical Gaussian process to regularize the homogenized contact problem of closing cracks.

Such smoothing function introduction has been physically justified by the analysis of micro-cracks opening and closure with the microscopic model. A homogenized cracks stress tensor, on which a perfect plastic model has been applied, to reproduce phenomena related to frictional sliding of cracks surfaces. Such modeling choices led to satisfying results, namely crescent-shaped and accurately positioned hysteresis loops. Quality of the resulting and identified model has been verified at the RVE scale and validated at the structural scale by the simulation of reinforced concrete building subject to earthquake loading. The simulation of the complete loading path has been achieved. This simulation served as a validation of the numerical robustness of the proposed continuum model. Improvements still need to be done regarding the quantification of energy dissipation at the local scale or structural level.

\section{REFERENCES}

[1] Bazant Z.P., Tabbara M., Kazemi M. and Pijaudier-Cabot G. 1990. Random particle model for fracture of aggregate and fibre composites. Journal of Engineering Mechanics, ASCE, 16(8):1686-1705.

[2] Herrmann H.J., Hansen A. and Roux S. 1989. Fracture of disordered, elastic lattices in two dimensions. Physical Review B, 39(1):637-648.

[3] Schlangen E. and Van Mier J. G. M. 1992. Experimental and numerical analysis of micromechanisms of fracture of cement-based composites. Cement and Concrete Composites, 14:105-118.

[4] D'Addetta G. A., Kun F. and Ramm E. 2002. On the application of a discrete model to the fracture process of cohesive granular materials. Granular Matter, 4:77-90.

[5] Delaplace A. and Desmorat R.. 2007. Discrete 3d model as complimentary numerical testing for anisotropic damage. Int J Fract, 148:115-128.

[6] Vassaux M., Richard B., Ragueneau F. \& Millard A. 2015. Lattice models applied to cyclic behavior description of quasi-brittle materials: advantages of implicit integration, Int. J. Num. \& Anal. Meth. In Geomechanics, 39:7, pp. 775-798.

[7] Schlangen E. and Garbozi E. J.. 1997. Fracture simulations of concrete using lattice models : computational aspects. Eng. Fracture Mech., 57: 319-332.

[8] Van Mier J. G. M., Van Vliet M. R. A., and Wang T. K. 2002. Fracture mechanisms in particle composites: statistical aspects in lattice type analysis. Mech. Mater, 34:705-724.

[9] Perkins E. and Williams J.R. 2001. A fast contact detection algorithm insensitive to 
object sizes, Engineering Computations, 18(1/2):48-62.

[10] O'Rourke J., Chien C.-B., Olso T. and Naddor D. 1982. A new linear algorithm for intersecting convex polygons. Computer Graphics and Image Processing, 19(4):384-391.

[11] Tillemans H.-J. and Herrmann H.J. 1995. Simulating deformations of granular solids under shear. Physica A: Statistical Mechanics and its Applications, 217(34):261-288.

[12] Morice E. 2014. Fissuration dans les matériaux quasi-fragiles : approche numérique et expérimentale pour la détermination d'un modèle incrémental à variables condensées. $\mathrm{PhD}$ thesis, Ecole Normale Supérieure de Cachan, France.

[13]Davie C.T. and Bicanic N. 2003. Failure criteria for quasi-brittle materials in lattice-type models. Commun. Numer. Meth. Engng, 19:703-713.

[14] Vassaux M., Ragueneau F., Richard B., and Millard A. 2014. Compressive behavior of a lattice discrete element model for quasi-brittle materials. Computational Modelling of Concrete Structures.

[15] Kotsovos, M.D. 1983. Effect of testing techniques on the post-ultimate behaviour of concrete in compression. Materiaux et construction, 16 (1):3-12.

[16]Fenwick RC and Paulay T. 1968. Mechanisms of shear resistance of concrete beams.

[17] Walraven, J.C. 1980. Aggregate interlock :a theoretical and experimental analysis. Thèse de doctorat de l'Université Technologique de Delft, The Netherlands.

[18] Gylltoft, K. 1984. A fracture mechanics model for fatigue in concrete, Matériaux et Construction ,17 (1) (1984) 55-58.

[19] Reinhardt H. W., Cornelissen H. A. W., Hordijk D. A. 1986. Tensile tests and failure analysis of concrete, Journal of Structural Engineering 112 (11); 24622477.

[20] Hordijk D. A. 1991. Local approach to fatigue of concrete, Ph.D. thesis, T.U. Delft, The Netherlands.
[21] Guyer R. A., McCall K. R., Boitnott G. N. 1995. Hysteresis, discrete memory, and nonlinear wave propagation in rock: A new paradigm, Physical review letters, 74 (17).

[22] Pensée V., Kondo D. and Dormieux L. 2002. Micromechanical analysis of anisotropic damage in brittle materials, Journal of Engineering Mechanics, 128 (8), pp. 889-897.

[23] Mertens S., Vantomme J. and Carmeliet J. 2007. Modelling of the influence of the damage on the behavior of concrete during tensile-compressive loading, Fracture mechanics of concrete structures.

[24] Simo J. C., Ju J. 1987. Strain and stress based continuum damage models formulation, International Journal of Solids and Structures, 23: 821-840.

[25] Meschke G., Lackner R. and Mang H. A. 1998. An anisotropic elastoplasticdamage model for plain concrete, International Journal for Numerical Methods in Engineering, 42 (4): 703727.

[27] Matallah M. and La Borderie C. 2009. Inelasticity-damage-based model for numerical modeling of concrete cracking, Engineering Fracture Mechanics 76 (8): 1087-1108.

[28] Sellier A., Casaux-Ginestet G., BuffoLacarrière L., Bourbon X. 2013. Orthotropic damage coupled with localized crack reclosure processing. Part i: Constitutive laws, Engineering Fracture Mechanics, 97: 148-167.

[29] Richard B. and Ragueneau F. 2013. Continuum damage mechanics based model for quasi brittle materials subjected to cyclic loadings: Formulation, numerical implementation and applications, Engineering Fracture Mechanics (98): 383-406.

[30] Richard B., Martinelli P., Voldoire F., Corus M., Chaudat T., Abouri S. and Bonfils N. 2015. Smart 2008: Shaking table tests on an asymmetrical reinforced concrete structure and seismic margins assessment, Engng Struct., 105:48-61. 